\title{
Comparing Health Gains, Costs and Cost- Effectiveness of 100s of Interventions in Australia and New Zealand: An Online Interactive League Table
}

Natalie Carvalho ( $\square$ natalie.carvalho@unimelb.edu.au )

The University of Melbourne https://orcid.org/0000-0002-5599-2209

\section{Tanara Sousa}

The University of Melbourne Faculty of Fine Arts and Music

\section{Anja Mizdrak}

University of Otago

Amanda Jones

University of Otago

Nick Wilson

University of Otago

\section{Tony Blakely}

University of Melbourne School of Population Health: The University of Melbourne School of Population and Global Health

\section{Research}

Keywords: cost-effectiveness, league table, priority-setting, costs, health gains

Posted Date: January 4th, 2022

DOI: https://doi.org/10.21203/rs.3.rs-1072911/v1

License: (c) (i) This work is licensed under a Creative Commons Attribution 4.0 International License. Read Full License 


\section{Abstract \\ Background}

This study compares the health gains, costs, and cost-effectiveness of hundreds of Australian and New Zealand (NZ) health interventions conducted with comparable methods in an online interactive league table designed to inform policy.

\section{Methods}

A literature review was conducted to identify peer-reviewed evaluations (2010 to 2018) arising from the Australia Cost-Effectiveness (ACE) research and NZ Burden of Disease Epidemiology, Equity and CostEffectiveness (BODE3) Programmes, or using similar methodology, with: health gains quantified as health-adjusted life years (HALYs); net health system costs and/or incremental cost-effectiveness ratio; time horizon of at least 10 years; and $3-5 \%$ discount rates.

\section{Results}

We identified 384 evaluations that met the inclusion criteria, covering 14 intervention domains: alcohol; cancer; cannabis; communicable disease; cardiovascular disease; diabetes; diet; injury; mental illness; other non-communicable disease; overweight and obesity; physical inactivity; salt; tobacco. There were large variations in health gain across evaluations: $33.9 \%$ gained less than $0.1 \mathrm{HALY}$ s per 1000 people in the total population over the remainder of their lifespan, through to $13.0 \%$ gaining $>10 \mathrm{HALY}$ s per 1000 people. Over a third (38.8\%) of evaluations were cost-saving.

\section{Conclusions}

League tables of comparably conducted evaluations illustrate the large health gain (and cost) variations per capita between interventions, in addition to cost-effectiveness. Further work can test the utility of this league table with policy makers and researchers.

\section{Background}

All jurisdictions have constraints on what preventive and other health services are provided, such as policy and public support, fixed health budgets or health sector capacity. Within these constraints, jurisdictions should aim to achieve maximal health benefits and, if possible, savings to future health expenditure - or at least a cost-effective return on investment, while accounting for other important criteria, including equity. Integrated epidemiological and economic evaluation studies provide estimates of future health gains, cost impacts, and cost-effectiveness of single or multiple interventions. If interventions fall below a pre-specified "willingness-to-pay (WTP) threshold", or are cost saving, they are 
considered good value for money and worthy of being introduced. However, the reliance on WTP thresholds alone to guide decision rules has been criticised, with the key problems identified being a lack of theoretical justification, the appropriate estimation of these thresholds, and the lack of accounting for other relevant decision-making considerations. $(1,2)$ For example, a fixed WTP threshold does not take budget considerations into account, and often interventions found to be "cost-effective" are not implemented due to budget impact constraints in that funding cycle.(3) On the health outcomes side, public health experts and policy makers are often unaware of the magnitude of potential health gains from interventions; comparable estimates of future health gains and costs impacts across multiple preventive and other health interventions are needed to inform the prioritisation alongside costeffectiveness.

One approach to prioritisation is league tables, pioneered by Alan William's comparison of cardiovascular disease treatments in 1985 to identify which treatment was near 'the top of the league'.(4) Such league tables typically rank health interventions by cost per life year or cost per quality-adjusted life year gained. League tables were quickly criticised about the lack of methodological consistency in conduct of costeffectiveness analysis (CEA), which could bias the ranking of interventions. $(5,6)$ Additionally, league tables may over-emphasise cost-effectiveness relative to the size of the health gain or cost-savings, addressing health inequalities, and intervention feasibility.(5)

We see an opportunity for a new era of league tables. First, there has been progress articulating best practice guidelines for the conduct and reporting of CEA (e.g. Consolidated Health Economic Evaluation Reporting Standards (CHEERS) checklist among others (7-9)), but also more generally in modelling the health impacts of preventive interventions.(10) Second, with the adoption of these guidelines, there are now higher-quality estimates from which to construct a more methodologically robust league table.(11) Third, online tools for presenting and interacting with data opens the potential for user input to league table generation, and greater flexibility in the presentation of output.

However, intervention simulation and cost-effectiveness studies often lack comparability. For example, variations in discount rates, time horizons over which benefits are assessed, and perspectives can lead to difficulties in making fair comparisons of health benefit, cost, and value for money. There are limited examples of league tables comparing interventions from methodologically consistent evaluations; for example: obesity interventions,(12,13) tobacco endgame strategies,(14) dietary sodium reduction interventions,(15) a varied package of preventive interventions,(16) and preventive and therapeutic interventions targeting non-communicable diseases.(17) Online repositories such as the Tufts New England CEA registry and Global Health CEA registry (www.cearegistry.org) contains thousands of costutility analyses conducted globally; however there are no restrictions on the comparability of methods used in the studies and therefore limited confidence in comparability.

Australia and New Zealand (NZ) are fortunate to now have a large body of evaluations (primarily preventive but some treatment) conducted mostly using a comparable proportional multi-state lifetable (PMSLT) method (18), spawned by the Assessing Cost-Effectiveness (ACE) studies in Australia - 
particularly the ACE-Prevention study (16) - and now also by the NZ Burden of Disease Epidemiology, Equity and Cost-Effectiveness Programme (BODE ${ }^{3}$; www.otago.ac.nz/bode3). This paper describes the collation of comparable evaluations from the $\mathrm{ACE}$ and $\mathrm{BODE}{ }^{3}$ Programmes, published or in press from 2010 to 2018. We provide outputs in comparable units of health outcomes, costs, and cost-effectiveness. Furthermore, we give graphical examples of league table comparisons using an online interactive tool, the Australia and NZ Health Intervention League Table (ANZ-HILT).

\section{Methods}

We compiled input data and health and economic outputs from similarly conducted evaluations from Australia and NZ, sourced from the following peer-reviewed publications:

- The Australian ACE Prevention Report,(16) with evaluations replaced by peer-reviewed paper if available.

- Australian peer-reviewed papers and reports using ACE methodology $(16,18)$ published from 1 January 2010 to 31 December 2018, found by searching on key authors (details in Appendix).

- NZ publications from the BODE ${ }^{3}$ Programme (www.otago.ac.nz/bode3).

The unit of presentation and analysis was an evaluation; a publication or report may include many evaluations of variants of the same intervention.

\section{Eligibility criteria}

Evaluations were included if they met these criteria:

- A clear description of the intervention duration and, where appropriate, frequency.

- Quantified health impacts in health-adjusted life years (HALYs): either quality-adjusted life years (QALYs) gained or disability-adjusted life years (DALYs) averted.

- Quantified either:

- at least two of the three following health system costs:

- Intervention cost

- Averted or incurred future health system cost offsets due to changing future disease incidence, both those related and unrelated to the diseases or conditions directly affected by the intervention. $(8,19)$

- Net costs (i.e. intervention costs minus cost offsets).

- an incremental cost-effectiveness ratio (ICER) - with the numerator satisfying the 'net cost' definition above.

- At least a 10-year time horizon post-intervention commencement for the accrual of HALYs and costs. 
- Applied a discount rate of between $3 \%$ and $5 \%$ for both health gains and costs.

\section{Data extracted from each evaluation}

We extracted the following data from each evaluation: (1) setting, including country, description and size of target population, year in which intervention started, currency and base-year for costs; (2) intervention characterisation, including duration of intervention and frequency of intervention, along with comparator; (3) methods, including time horizon of simulation follow-up and discount rate; (4) outcomes, including HALYs gained, costs, ICER, and 95\% uncertainty intervals.

In cases where data were not explicitly provided in the main paper or supplemental materials, we estimated the total eligible target population and calculated the ICER from net costs and HALYs.

\section{Processing of extracted outputs and visualisation of evaluations}

Evaluations were categorised into one of 14 domains derived from the Global Burden of Disease Study's risk factor domains: alcohol; cancer; cannabis; communicable disease; cardiovascular disease (CVD); diabetes; diet; injury; mental illness; other non-communicable disease (NCD); overweight and obesity; physical inactivity; salt (dietary); and tobacco.

Based on what population level the intervention was directed toward, each intervention was assigned to one of three categories: population-wide, intermediary 'partial targeting', or 'targeted'. Population-wide represents interventions such as the reformulation of food and tobacco taxes. Partial targeting was any programme directed toward less than a quarter of the total population, such as school-based programmes, and captured most screening programmes and vaccination programmes. Targeted was any treatment intervention for people with a disease. This included rehabilitation and screening postdiagnosis but excluded secondary prevention among people with risk factors rather than a disease (e.g. hypertension or obesity; coded as 'partial targeted').

\section{An online, user-friendly visualisation tool}

To assist interpretation and make evaluations flexibly available to interested users, we created a userfriendly R Shiny App tool (ANZ-HILT) to allow visualisation of evaluations (https://leaguetable.shinyapps.io/bode3/). ANZ-HILT allows interventions to be compared by HALYs gained, net costs and ICER (where the intervention is not cost-saving). Interventions were categorised as 'cost-saving' if net costs were negative and HALYs positive, and as 'dominated' if both the net costs were positive and HALYs negative, compared to the comparator scenario. HALYs and costs are shown as a total applied to the whole country and per 1000 people in the total population in the base-year. Costs are presented inflation 
and purchasing power parity (Organisation for Economic Co-operation and Development (OECD)) adjusted to any year between 2010 and 2016 in three currencies, United States dollars (US\$), NZ dollars (NZ\$) or Australian dollars (AU\$). In the current paper we give graphical examples of league table comparisons using this tool to showcase some of the possible comparisons across interventions. ANZHILT also contains additional evaluations and outputs beyond the scope of this paper.

\section{Results}

A total of 384 evaluations met the inclusion criteria (see Appendix). Of the 318 Australian evaluations, 94 were reported in the original ACE-Prevention Report and also published in a journal article (used as the primary source), 137 were published only in the Report. Beyond the original ACE-Prevention Report, 15 more evaluations arose from an obesity report and another 72 arose from 23 peer-reviewed articles. All 66 NZ evaluations came from 25 peer-reviewed articles arising from the BODE ${ }^{3}$ Programme.

We excluded three interventions deemed no longer relevant: circumcision of men to prevent HIV infection (not relevant in NZ and Australia),(16) and two cardiovascular disease polypill evaluations with a price of AU\$5000 per year(16) - which is far higher than current pricing.

\section{Characteristics of included evaluations}

Table 1 shows the characteristics of evaluations, by country. The majority of Australian evaluations were published before 2015 (77.7\%) and used 2003 as the base-year, while the majority of NZ evaluations were published after 2015 (92.4\%) and all used 2011 as the base-year. All NZ evaluations used a lifetime perspective, and only $4.1 \%$ of the Australian evaluations had less than a lifetime perspective - and these were mostly mental health and communicable diseases interventions. All evaluations used a $3 \%$ discount rate. The majority of Australian evaluations were targeted (8.2\%) or partially targeted (56.6\%), while the NZ evaluations were mostly population-wide (72.7\%). The large majority of evaluations in both countries $(92.4 \%)$ were preventive. Over half $(57.3 \%)$ of evaluations across both countries were for interventions that persisted over the remainder of the population's life span (e.g. tax interventions) with the second most common intervention duration being one-off or up to 1 year (20.8\% overall). Over half of Australian evaluations were related to cardiovascular disease (29.6\%), overweight \& obesity (14.2\%) and diet (13.5\%). The NZ evaluations were more concentrated by domain, with $81.8 \%$ being from one of three domains (cancer, dietary salt and tobacco). 
Table 1

Characteristics of included evaluations

2015-2018

\section{Base-year in model}

2000-04

2005-09

2010-15

Time horizon

$10 y$ to <lifetime

Lifetime

Discount rate (annual)

$3 \%$

Other

Degree of targeting

Population-wide

Partially targeted

Targeted

Intervention duration

One-off or up to 1 year

1-5 years

6-20 years

*2016 US\$

† Most studies reported results for HALYs and costs for either a total population perspective (e.g. for all eligible people in Australia) or a per capita perspective - but not both (although we were able to sometimes calculated both if sufficient data was reported in the paper).
Australia

$\mathrm{N} \quad \%$

318

247

71

New Zealand

$\%$

66

384

$252 \quad 65.6 \%$

$132 \quad 34.4 \%$

$\begin{array}{lllll}22.3 \% & 61 & 92.4 \% & 132 & 34.4 \%\end{array}$

207

$65.1 \%$

0

$0 \%$

$207 \quad 53.9 \%$

40

$12.6 \%$

0

$0 \%$

$40 \quad 10.4 \%$

71

$22.3 \%$

66

$100 \%$

137

$35.7 \%$

13

$4.1 \%$

0

$0 \%$

13

$3.4 \%$

95

$95.9 \%$

66

$100 \%$

$371 \quad 96.6 \%$

318

$100 \%$

66

$100 \%$

384

$0 \%$

0

$0 \%$

$0 \quad 0 \%$

112

$35.2 \%$

48

$72.7 \%$

160

$41.7 \%$

180

$56.6 \%$

14

$21.2 \%$

194

$50.5 \%$

26

$8.2 \%$

4

$6.1 \%$

30

$7.8 \%$

\begin{tabular}{llllll}
64 & $20.1 \%$ & 16 & $24.2 \%$ & 80 & $20.8 \%$ \\
\hline 9 & $2.8 \%$ & 1 & $1.5 \%$ & 10 & $2.6 \%$ \\
\hline 20 & $6.3 \%$ & 1 & $1.5 \%$ & 21 & $5.5 \%$
\end{tabular}




\begin{tabular}{|c|c|c|c|c|c|c|}
\hline \multirow[b]{2}{*}{ Persistent } & \multicolumn{2}{|c|}{ Australia } & \multicolumn{2}{|c|}{ New Zealand } & \multicolumn{2}{|c|}{ Total } \\
\hline & 172 & $54.3 \%$ & 48 & $72.7 \%$ & 220 & $57.3 \%$ \\
\hline Not specified & 53 & $16.7 \%$ & 0 & $0 \%$ & 53 & $13.8 \%$ \\
\hline \multicolumn{7}{|l|}{ Type of intervention } \\
\hline Prevention & 298 & $93.7 \%$ & 57 & $86.4 \%$ & 355 & $92.4 \%$ \\
\hline Treatment & 20 & $6.3 \%$ & 4 & $6.1 \%$ & 24 & $6.3 \%$ \\
\hline Missing & 0 & $0 \%$ & 5 & $7.6 \%$ & 5 & $1.3 \%$ \\
\hline \multicolumn{7}{|l|}{ Domain } \\
\hline Cancer & 27 & $8.5 \%$ & 8 & $12.1 \%$ & 35 & $9.1 \%$ \\
\hline Alcohol & 16 & $5.0 \%$ & 0 & $0 \%$ & 16 & $4.2 \%$ \\
\hline Cannabis or other illicit drugs & 5 & $1.6 \%$ & 0 & $0 \%$ & 5 & $1.6 \%$ \\
\hline Communicable disease & 7 & $2.2 \%$ & 5 & $7.6 \%$ & 12 & $3.1 \%$ \\
\hline Cardiovascular disease & 94 & $29.6 \%$ & 1 & $1.5 \%$ & 95 & $24.7 \%$ \\
\hline Diabetes & 13 & $4.1 \%$ & 0 & $0 \%$ & 13 & $3.4 \%$ \\
\hline Diet & 43 & $13.5 \%$ & 0 & $0 \%$ & 43 & $11.2 \%$ \\
\hline Injury & 1 & $0.3 \%$ & 5 & $7.6 \%$ & 6 & $1.6 \%$ \\
\hline Mental illness & 8 & $2.5 \%$ & 0 & $0 \%$ & 8 & $2.1 \%$ \\
\hline Other NCD & 26 & $8.2 \%$ & 0 & $0 \%$ & 26 & $6.8 \%$ \\
\hline Overweight \& obesity & 45 & $14.2 \%$ & 1 & $1.5 \%$ & 46 & $12.0 \%$ \\
\hline Physical activity & 16 & $5.0 \%$ & 0 & $0 \%$ & 16 & $4.2 \%$ \\
\hline Salt (dietary) & 3 & $0.9 \%$ & 32 & $48.5 \%$ & 35 & $9.1 \%$ \\
\hline Tobacco & 14 & $4.4 \%$ & 14 & $21.2 \%$ & 28 & $7.3 \%$ \\
\hline \multicolumn{7}{|l|}{ HEALTH GAIN } \\
\hline \multicolumn{7}{|l|}{ HALYs per 1000 total population } \\
\hline$<0.10$ & 122 & $28.4 \%$ & 8 & $12.1 \%$ & 130 & $33.9 \%$ \\
\hline $0.10-1$ & 86 & $27.0 \%$ & 2 & $3.0 \%$ & 88 & $22.9 \%$ \\
\hline \multicolumn{7}{|l|}{ *2016 US\$ } \\
\hline $\begin{array}{l}\text { † Most studies reported results } \\
\text { all eligible people in Australia) } \\
\text { sometimes calculated both if s }\end{array}$ & $\begin{array}{l}\text { and } \\
\text { pita } p \\
\text { ata w }\end{array}$ & $\begin{array}{l}\text { ts for eit } \\
\text { pective } \\
\text { reported }\end{array}$ & a to & $\begin{array}{l}\text { opulati } \\
\text { th (alth }\end{array}$ & rspec & $\begin{array}{l}\text { (e.g. for } \\
\text { able to }\end{array}$ \\
\hline
\end{tabular}




\begin{tabular}{|c|c|c|c|c|c|c|}
\hline \multirow[b]{2}{*}{$1-10$} & \multicolumn{2}{|c|}{ Australia } & \multicolumn{2}{|c|}{ New Zealand } & \multicolumn{2}{|c|}{ Total } \\
\hline & 77 & $24.2 \%$ & 30 & $45.5 \%$ & 107 & $27.9 \%$ \\
\hline$>10$ & 28 & $8.8 \%$ & 22 & $33.3 \%$ & 50 & $13.0 \%$ \\
\hline Missing $^{\dagger}$ & 5 & $1.6 \%$ & 4 & $6.1 \%$ & 9 & $2.3 \%$ \\
\hline \multicolumn{7}{|c|}{ HALYs per person in target population } \\
\hline$<01$ & 28 & $8.8 \%$ & 23 & $34.9 \%$ & 51 & $13.3 \%$ \\
\hline 01-099 & 0 & $0 \%$ & 20 & $30.3 \%$ & 20 & $5.2 \%$ \\
\hline $0.1-0.99$ & 1 & $0.3 \%$ & 3 & $4.5 \%$ & 4 & $1.0 \%$ \\
\hline Missing $^{\dagger}$ & 289 & $90.9 \%$ & 20 & $30.3 \%$ & 309 & $80.5 \%$ \\
\hline \multicolumn{7}{|c|}{ INCREMENTAL HEALTH EXPENDITURE } \\
\hline \multicolumn{7}{|c|}{ Net cost* per 1000 total population } \\
\hline < US\$0 [Cost saving] & 103 & $32.4 \%$ & 46 & $69.7 \%$ & 149 & $38.8 \%$ \\
\hline US\$0 to $\$ 10,000$ & 99 & $31.2 \%$ & 9 & $13.6 \%$ & 108 & $28.1 \%$ \\
\hline Cost > US\$10,000 & 67 & $21.1 \%$ & 8 & $12.1 \%$ & 75 & $19.5 \%$ \\
\hline Missing $^{\dagger}$ & 49 & $15.4 \%$ & 3 & $4.6 \%$ & 52 & $13.5 \%$ \\
\hline \multicolumn{7}{|c|}{ Net cost* per target population } \\
\hline < US\$0 [Cost saving] & 1 & $0.3 \%$ & 32 & $48.5 \%$ & 33 & $8.6 \%$ \\
\hline US\$0 to $\$ 1000$ & 24 & $7.6 \%$ & 10 & $15.2 \%$ & 34 & $8.9 \%$ \\
\hline Cost > US\$1000 & 4 & $1.3 \%$ & 2 & $3.0 \%$ & 6 & $1.6 \%$ \\
\hline Missing $^{\dagger}$ & 289 & $90.9 \%$ & 22 & $33.3 \%$ & 311 & $81.0 \%$ \\
\hline \multicolumn{7}{|c|}{ COST per HALY or Incremental cost-effectiveness ratio } \\
\hline Cost saving & 97 & $30.5 \%$ & 47 & $71.2 \%$ & 144 & $37.5 \%$ \\
\hline US\$0 to $\$ 50,000$ per HALY & 127 & $39.9 \%$ & 17 & $25.8 \%$ & 144 & $37.5 \%$ \\
\hline$>$ US\$50,000 per HALY & 82 & $25.8 \%$ & 1 & $1.5 \%$ & 83 & $21.6 \%$ \\
\hline Dominated & 3 & $0.9 \%$ & 0 & $0 \%$ & 3 & $0.8 \%$ \\
\hline
\end{tabular}

*2016 US\$

† Most studies reported results for HALYs and costs for either a total population perspective (e.g. for all eligible people in Australia) or a per capita perspective - but not both (although we were able to sometimes calculated both if sufficient data was reported in the paper). 


\begin{tabular}{|c|c|c|c|c|c|c|}
\hline \multirow[b]{2}{*}{ Missing $^{\dagger}$} & \multicolumn{2}{|c|}{ Australia } & \multicolumn{2}{|c|}{ New Zealand } & \multicolumn{2}{|c|}{ Total } \\
\hline & 9 & $2.8 \%$ & 1 & $1.5 \%$ & 10 & $2.6 \%$ \\
\hline \multicolumn{7}{|c|}{ ^2016 US\$ } \\
\hline \multicolumn{7}{|c|}{$\begin{array}{l}\text { † Most studies reported results for HALYs and costs for either a total population perspective (e.g. for } \\
\text { all eligible people in Australia) or a per capita perspective - but not both (although we were able to } \\
\text { sometimes calculated both if sufficient data was reported in the paper). }\end{array}$} \\
\hline
\end{tabular}

The majority of evaluations across both countries (97.7\%) were able to have results expressed for the total population, whereas only a minority (19.5\%) were able to have results expressed per capita of a target population. The distribution of HALYs gained and net costs per 1000 of the total population, and per capita of the target population, are shown in Table 1. Most (71.2\%) of the NZ evaluations were costsaving, but only $30.5 \%$ of the Australian ones were. Conversely, $26.7 \%$ of the Australian evaluations had either an ICER >US\$50,000 (beyond the rule of thumb of Gross Domestic Product (GDP) per capita being a threshold beyond which interventions are deemed not cost-effective) or were dominated (i.e. performed worse than the comparator), compared to only $1.5 \%$ of the NZ evaluations.

\section{Selected examples of ANZ-HILT outputs}

Figure 1 demonstrates a histogram output from ANZ-HILT showcasing a selection of interventions. The left-hand panel of ANZ-HILT allows the user to select permutations of: domain (three here: cancer, diet and tobacco), actual interventions (seven here), currency and year to show dollars in, range of publication years that the evaluation was published in, and the outcome variable to plot (HALYs here). The tabs: across the top allow the user to view instructions, population denominator (here per 1000 of the total population), or cost-effectiveness plane (see Figure 2 below); and beneath toggle between table and 'plot top 10' options (plot shown here). A hover-over with one's computer mouse allows the user to see the following for each evaluation: expected number of HALYs / discount rate / time horizon of follow-up / comparator / and intervention duration and/or frequency. For example, for the $10 \%$ per annum tobacco tax intervention, hovering over the bar will cause the following text to appear: " 53,200 / 0.03 / Lifetime / Business-as-usual (no tax increases from 2011-to 2025) / 14 years of tax increases, then persistent". Further information can be found in the table tab, e.g. the actual lower and upper uncertainty limits.

Turning to the substantive patterns in Figure 1, there is an over 1000-fold variation from 23 per 1000 (95\% uncertainty interval (UI): 18 to 29) HALYs gained over the remainder of life for NZ population aged 35+ years in 2011 for $25 \%$ of salt in processed food being replaced by potassium and magnesium salts (where that intervention is 'left on' for the remainder of the population's lifespan), down to 0.019 per 1000 ( $95 \%$ UI: 0.011 to 0.029 ) for all stage III colon cancer patients diagnosed in 2011 being assisted by cancer care coordinators to navigate more quickly and with higher coverage to surgery and chemotherapy. With this (and other intervention combinations), differences in the timespan and target groups of interventions often account for differences in the magnitude of health gains. 
This variability in intervention conceptualisation noted, we can make some inferences from the selected examples shown Figure 1:

- $25 \%$ salt substitution with potassium and magnesium salts (NZ) and food taxes across saturated fat, excess salt, sugar-sweetened beverages and sugar in processed foods (Australia) leads to similarly large health gains.

- Interventions such as 'Tick' logos on health food and dietary advice for those with high blood pressure have much smaller health gains when summed up across the population.

The health system expenditure impacts of the interventions shown in Figure 1 are correlated - but with large cost-savings (due to future disease rates being considerably lower) for interventions with large health gains (Supplementary Figure 1). Intervention impacts can also be presented in terms of HALYs gained per capita in the target population (Supplementary Figure 2).

Figure 2 is an example of a cost-effectiveness plane output for five evaluations, with two overlays: the text pop-ups that appear as the user hovers over each point are shown for all five interventions; the black dashed line is a super-imposed threshold line at about GDP per capita per HALY. The plane now allows the user to simultaneously see (often massive) variation between interventions in all of health gain, cost and cost per HALY gained. For this example, we present results in 2016 US\$. For example, the tobacco retail outlet reduction intervention is in the southeast quadrant with substantial health gains (7 HALYs per 1000 over the remaining lifespan of the population) and cost-savings (US\$89,100 per 1000). On effectiveness and efficiency grounds alone, this intervention should be considered for implementation but there are other considerations such a political will and societal preferences that are not captured in ANZ-HILT. The Helicobacter pylori screening programme (to detect infection that is then treated with antibiotics, reducing stomach cancer incidence rates years into the future) is in the northeast quadrant, but beneath the willingness to pay line (black dashed line) suggesting it is cost-effective at a GDP per capita threshold. The computed tomography (CT) screening of heavy smokers is also in the northeast quadrant costing US\$42,000 per QALY gained (i.e. 33.9/0.81) - about the threshold GDP per capita per QALY gained.

\section{Discussion}

This paper demonstrates the consolidation of key health and economic outputs from hundreds of comparably conducted evaluations for Australia and NZ in a world first online interactive league table. ANZ-HILT allows for the comparison of health gain, net cost and cost-effectiveness of (mostly) preventive interventions across a wide range of domains. The league table discloses large differences in impact between interventions. Such information has hitherto not been easily accessible by stakeholders and endusers. Our aim is to better inform policy makers and health experts, who are often unaware of the relative magnitude of intervention impact.

\section{Early indications of benefits and barriers for end-users}

Page $11 / 18$ 
We have some evidence of utility and impact to date. First, a league table of dietary interventions was the backbone to discussions requested by the NZ Minister of Health on food reformulation options, as he was in parallel in negotiation with the food industry. Second, key informant interviews of 16 senior policy makers in Australia by an independent consult (commissioned by us as part of other work, February to March 2020, unpublished) found strong support for greater information of the type shown in ANZ-HILT: "There was general agreement that the lack of robust, comparable, and easy-to-access data on the impacts of various health programs hinders the design and prioritisation of public health interventions". However, there were also barriers to uptake identified, including a culture in policy making that was not always receptive to such evidence (e.g. due to time, capacity or other reasons), that will require addressing beyond the simple publication of a tool such as ANZ-HILT (e.g. outreach and championing).

\section{Strengths and Limitations}

The strengths of ANZ-HILT include the selection of evaluations meeting comparability criteria, and visualisations through graphs that focus not only on incremental cost-effectiveness - but also net heath gains and costs. There are also many limitations. First, we can only include what has been evaluated. It would be a false conclusion that because dietary interventions in ANZ-HILT tend to have lower health gains per 1000 population that we expect all dietary interventions to be similar; it depends on what specific evaluation researchers chose. Second, we use explicit criteria to select comparable evaluations, but we do not further restrict based on the quality of evaluation. Rather, we necessarily use a caveat emptor or 'user beware' approach - facilitated by provision of URL links to the underlying published evaluations. Third, the coding of some of the data extracted from evaluations is imprecise, most notably whether the intervention was population-wide or partially targeted. Regarding targeting, while we regard the presentation of HALYs and net costs per capita is a useful goal and part of the functionality in ANZHILT, determining the correct 'target' population is often challenging. For example, is the target population for a smoking cessation programme: all smokers, just those who are open to the idea of quitting, or only those planning a quit attempt? Lastly, there are considerations beyond health benefits, costs and costeffectiveness that are not included in ANZ-HILT, yet that may be relevant to policymakers including equity impacts of interventions.

\section{Conclusions}

League tables will never provide all the information necessary for policymakers to make prioritisation decisions, but they could be an excellent starting point for deliberation. Further research could probe validity (e.g. age-standardisation of outputs, comparability of costing methods) and quality (e.g. CHEERS checklist); extend outputs (e.g. health gains and net costs in first 10 and 10-20 years post-intervention); include compatible evaluations from other countries; and further research utility and impact with end users including policy makers and researchers. We propose that other researchers and countries consider contributing to a global-HILT.

\section{List Of Abbreviations}


ACE: Australia Cost-Effectiveness

ANZ-HILT: Australia and NZ Health Intervention League Table

AUȘ: Australian dollars

BODE3: Burden of Disease Epidemiology, Equity and Cost-Effectiveness

CHEERS: Consolidated Health Economic Evaluation Reporting Standards

CEA: Cost-effectiveness analysis

CT: Computed tomography

CVD: Cardiovascular disease

GDP: Gross Domestic Product

HALYs: Health-adjusted life years

ICER: Incremental cost-effectiveness ratio

NCD: non-communicable disease

NZ: New Zealand

NZ\$: NZ dollars

OECD: Organisation for Economic Co-operation and Development

PMSLT: Proportional multi-state lifetable

US\$: United States dollars

WTP: Willingness-to-pay

\section{Declarations}

Ethics approval and consent to participate:

Not applicable.

Consent for publication:

Not applicable.

Availability of data and materials: 
All data generated and analysed during this study are available from the corresponding author upon request.

\section{Competing interest statements:}

All authors have no conflicts of interest to declare.

\section{Funding:}

Funding was received from The Health Research Council of New Zealand (16/443); The New Zealand Ministry of Business, Innovation and Employment (UOOX1406); The Australian Institute of Health and Welfare (TA37980). NC received a University of Melbourne McKenzie Postdoctoral Fellowship.

\section{Authors' contributions:}

TB and NW conceptualised the study. TVS, AM, AJ, NW and TB compiled the online league table database. TB, TVS, NW and NC drafted the manuscript, and all authors contributed towards critical revisions for content. TB, TVS and NC have verified the underlying data. All authors approved the final version to be published.

\section{Acknowledgments:}

We thank Drs Cristina Cleghorn, Nhung Nghiem and Linda Cobiac for comments on earlier drafts of this paper.

\section{References}

1. Marseille E, Larson B, Kazi DS, Kahn JG, Rosen S. Thresholds for the cost-effectiveness of interventions: alternative approaches. Bull World Health Organ. 2015;93(2):118-24. Epub 2015/04/18.

2. Bertram MY, Lauer JA, De Joncheere K, Edejer T, Hutubessy R, Kieny MP, et al. Cost-effectiveness thresholds: pros and cons. Bull World Health Organ. 2016;94(12):925-30. Epub 2016/12/21.

3. Bilinski A, Neumann P, Cohen J, Thorat T, McDaniel K, Salomon JA. When cost-effective interventions are unaffordable: Integrating cost-effectiveness and budget impact in priority setting for global health programs. PLoS Med. 2017;14(10):e1002397. Epub 2017/10/03.

4. Williams A. Economics of coronary artery bypass grafting. Br Med J (Clin Res Ed). 1985;261(6491):326-9.

5. Mason J, Drummond M, Torrance G. Some guidelines on the use of cost effectiveness league tables. BMJ (Clinical research ed). 1993;306(6877):570-2. Epub 1993/02/27.

6. Drummond M, Torrance G, Mason J. Cost-effectiveness league tables: more harm than good? Social science \& medicine (1982). 1993;37(1):33-40. Epub 1993/07/01. 
7. Caro JJ, Briggs AH, Siebert U, Kuntz KM. Modeling Good Research Practices-Overview: A Report of the ISPOR-SMDM Modeling Good Research Practices Task Force-1. Value in Health. 2012;15(6):796803.

8. Sanders GD, Neumann PJ, Basu A, et al. Recommendations for conduct, methodological practices, and reporting of cost-effectiveness analyses: Second panel on cost-effectiveness in health and medicine. JAMA. 2016;316(10):1093-103.

9. Husereau D, Drummond M, Petrou S, Carswell C, Moher D, Greenberg D, et al. Consolidated Health Economic Evaluation Reporting Standards (CHEERS)-explanation and elaboration: a report of the ISPOR Health Economic Evaluation Publication Guidelines Good Reporting Practices Task Force. Value in Health. 2013;16(2):231-50. Epub 2013/03/30.

10. Briggs ADM, Wolstenholme J, Blakely T, Scarborough P. Choosing an epidemiological model structure for the economic evaluation of non-communicable disease public health interventions. Population Health Metrics. 2016;14(17).

11. Mason JM. Cost-per-QALY league tables: their role in pharmacoeconomic analysis. PharmacoEconomics. 1994;5(6):472-81. Epub 1994/05/08.

12. Ananthapavan J, Sacks G, Brown V, Moodie M, Nguyen P, Barendregt J, et al. Assessing CostEffectiveness of Obesity Prevention Policies in Australia 2018 (ACE-Obesity Policy). Melbourne,. Australia: Deakin University, 2018.

13. Cecchini M, Sassi F, Lauer JA, Lee Y, Y, Guajardo-Barron V, Chisholm D. Tackling of unhealthy diets, physical inactivity, and obesity: health effects and cost-effectiveness. The Lancet. 2010;376(9754):1775-84.

14. van der Deen FS, Wilson N, Cleghorn C, Kvizhinadze G, Cobiac L, Nghiem N, et al. Impact of five tobacco endgame strategies on future smoking prevalence, population health and health system costs: two modelling studies to inform the tobacco endgame. Tob Control. 2017;27(3):278-86.

15. Nghiem N, Blakely T, Cobiac LJ, Cleghorn CL, Wilson N. The health gains and cost savings of dietary salt reduction interventions, with equity and age distributional aspects. BMC Public Health. 2016;16(1):423.

16. Vos T, Carter R, Barendregt J, Mihalopoulis C, Veerman L, Magnus A, et al. Assessing CostEffectiveness in the Prevention (Ace-Prevention): Final Report. University of Queensland and Deakin University, 2010 September 2010. Report No.

17. Salomon JA, Carvalho N, Gutierrez-Delgado C, Orozco R, Mancuso A, Hogan DR, et al. Intervention strategies to reduce the burden of non-communicable diseases in Mexico: cost effectiveness analysis. BMJ (Clinical research ed). 2012;344:e355.

18. Blakely T, Moss R, Collins J, Mizdrak A, Singh A, Carvalho N, et al. Proportional multistate lifetable modelling of preventive interventions: concepts, code and worked examples. Int J Epidemiol. 2020;49(5):1624-36. Epub 2020/10/11.

\section{Figures}




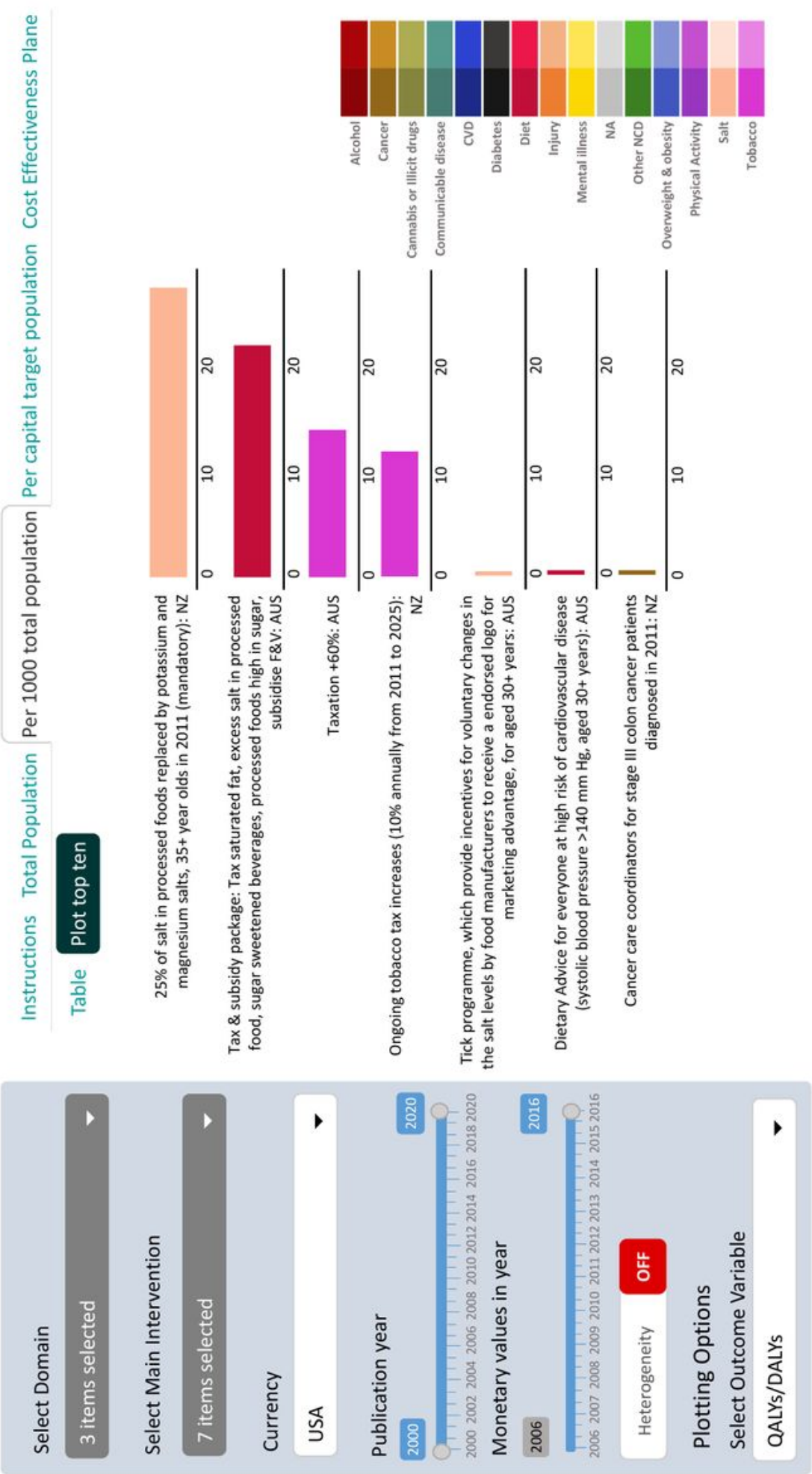

Figure 1

HALYs gained per 1000 in the total population, for selected Australian and New Zealand health sector interventions 

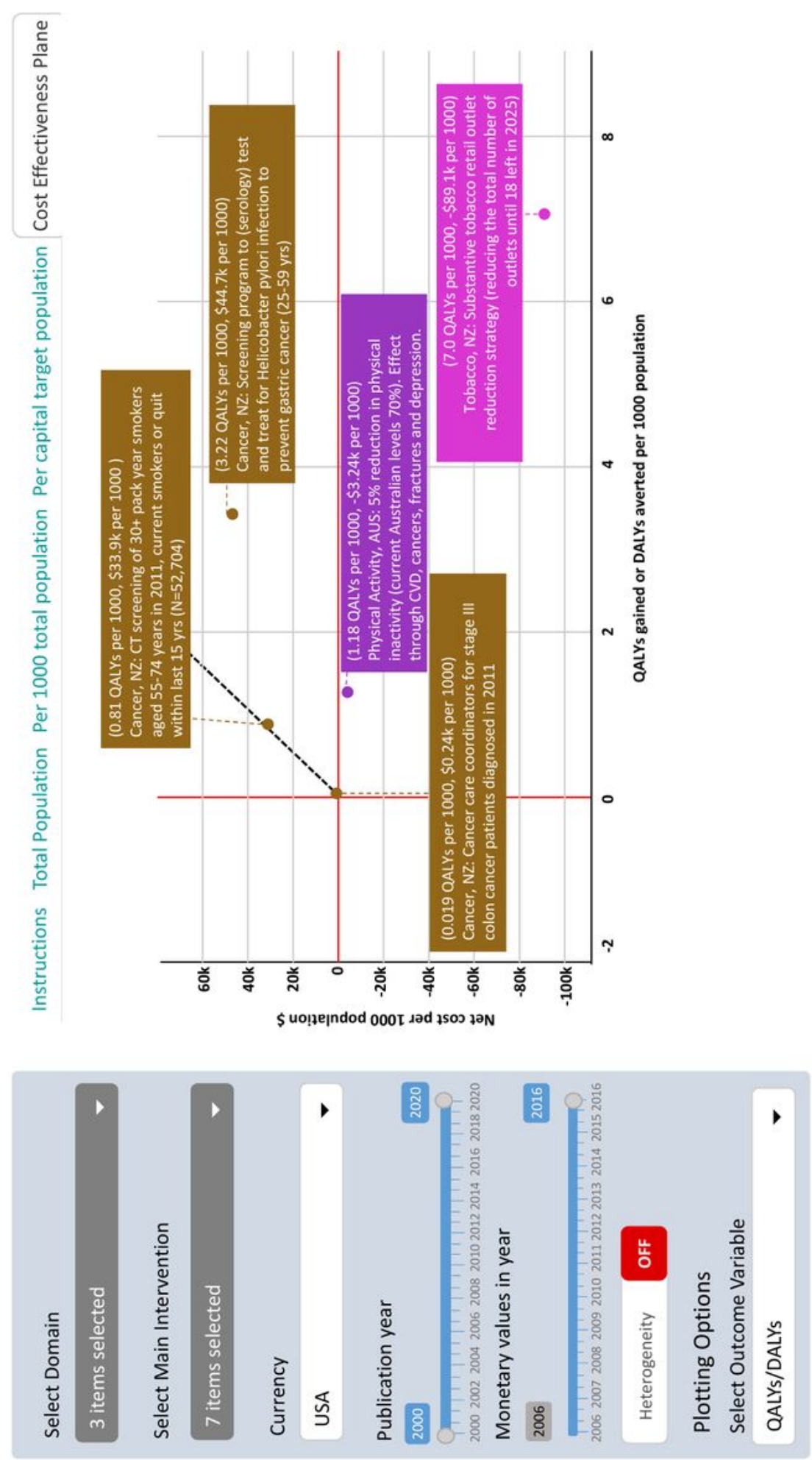

Figure 2

Cost-effectiveness plane for selected interventions

\section{Supplementary Files}

This is a list of supplementary files associated with this preprint. Click to download. 
- BODE3LeagueTableAppendix.docx 